\title{
Volume ratio of two immiscible phases measured by electrical impedance tomography
}

\author{
Michal Malík ${ }^{1, *}$, Jiři Primas ${ }^{1}$, Michal Kotek ${ }^{2}$, Václav Kopecký ${ }^{2}$, Bohuš Kysela ${ }^{3}$ and Radek \\ $\breve{S u l c}^{3}$ \\ ${ }^{1}$ Institute of Novel Technologies and Applied Informatics, Faculty of Mechatronics, Informatics and \\ Interdisciplinary Studies, Technical University of Liberec, Czech Republic \\ ${ }^{2}$ Department of Physical Measurements at the Institute for Nanomaterials, Advanced Technology and \\ Innovation at the Technical University of Liberec, Czech Republic \\ ${ }^{3}$ Department of Process Engineering at the Faculty of Mechanical Engineering, Czech Technical \\ University in Prague
}

\begin{abstract}
The mixing of two immiscible phases is a process commonly seen in many branches of industry. Whether it be desirable (e.g. mixing of ingredients in chemical or food industry) or undesirable (e.g. sediments or contaminants in water purification) process, it has to be taken into consideration and a detailed description would be beneficial to any end product. This paper deals with a method for observing the volume ratio of two immiscible phases from the state of total separation, during the mixing process until the state of a homogenous mixture using industrial Electrical Impedance Tomography (EIT) system ITS p2+. The paper also shows a great agreement between the data obtained through measurement using this method and data derived from a theoretical formula. Also using EIT the authors were able to provide additional information describing the mixing process in real time.
\end{abstract}

\section{Introduction}

In a vast amount of industrial applications (water treatment, energetics, chemical engineering, etc.) there is some form of mixing of different phases involved. In case of deliberate mixing the end goal of this process is usually reaching a homogeneous mixture with the minimum expenditure of energy and time. In case of unwanted mixing we need to know the volume of the second phase or the state of the mixture. Both cases benefit from observation or measuring the process for the purpose of determining if and how the end goal has been reached.

This paper focuses on the use of Electrical Impedance Tomography (EIT) as a measuring tool for the use in mixing, where it is not possible to use various other methods (e.g. ultrasonic measuring methods, capacitance measurements or optical methods such as image processing, particle image velocimetry, interferometric particle imaging, etc.). Since optical measuring methods are the most commonly used, the main advantage of using EIT becomes evident if the mixed media or the container is optically non-transparent.

\footnotetext{
* Corresponding author: michal.malik@,tul.cz
} 
The principle behind the Electrical Impedance Tomography is measuring the conductivity/impedance of a medium between two sets of electrodes [1]. As with all tomography methods this is done over a whole measuring plane by using several sets of electrodes in the same plane and switching between them. Since unwanted electrochemical effects may occur in direct current measurements, the whole EIT measurement is done using alternating currents in the range of milliampers at tens of $\mathrm{kHz}$. Mathematically, the problem of reconstructing conductivity from surface measurements of current and potential is a nonlinear inverse problem and is severely ill-posed. The detailed mathematical formulation of this problem has been stated by Alberto Calderón [2]. This means, the vast amount of measured data have to be processed by a suitable modelling software with the results usually displayed in the form of scalar maps. These show the planar conductivity distribution in the interrogated area.

This principle has several advantages over other methods usable for the same purpose. Mainly, we can see "inside" a lot of processes, but the method does not require us to introduce any kind of ionizing radiation or foreign agents for tracing purposes. This is a huge advantage in medicine, where EIT is widely used - e. g. lung and breast EIT diagnostics. Another very important advantage is that EIT also does not require any probe or other physical object to be inserted into the interrogation area. The only requirement for the electrodes of the tomography system is to have a conductive contact with the medium. They can thus be on the sides of the container/pipe, where they merge with the surface of the container and do not intrude into the medium itself. This also means that the use of EIT does not affect the character of flow in the measured system.

It can be said that nearly all ordinary chemical substances have different conductivities, which mainly depend on free ion content [3]. Thus EIT allows us to observe the course of their mixing accurately and with ease from the initial state, when the substances are separate, to the final state, when a homogenous ideal mixture is achieved.

One such common problem is to describe the mixing of a conductive medium with a nonconductive one. The theory behind the conductivity of such a mixture is well known [4]. However to be able to analyse the mixture using the theoretical description, we need it in its homogenous state. Here is the point, when the EIT system comes into play, since it is a very suitable tool for ascertaining if the mixture has reached homogeneity. This is due to its capability of showing a planar (or even spatial) distribution of conductivity. If during the mixing process the measured conductivities in each point of the measured plane (volume) reach the same value, we can consider the mixture to be homogenous. This allows us to characterise the mixture by this single conductivity value, which we can use to represent it in the theoretical description.

There are many ways of calculating the conductivity of a two-part mixture with known original conductivities. In [4] it has been shown that the volume ratio of two mixed substances, where the first one has a known conductivity and the second one is nonconductive, can be calculated by the means of the following formula:

$$
\varepsilon=\frac{2-\frac{2 \gamma}{\gamma_{0}}}{2+\frac{\gamma}{\gamma_{0}}}
$$

where $\gamma$ is the conductivity of the final homogenous mixture, $\gamma_{0}$ is the conductivity of the conductive substance and $\varepsilon$ is the volume ratio of both mixed substances and can be defined as follows:

$$
\varepsilon=\frac{V_{1}}{V_{0}},
$$

where $V_{l}$ is the volume of non-conductive substance and $V_{0}$ is the volume of the conductive substance in the mixture. 
Using EIT we are able to monitor the time development of the mixture conductivity and we can easily find the point, when the conductivity remains constant. Then we can be sure that the mixture is homogenous and the measured conductivity value is the right one.

\section{Experiment methodology}

In our experiments we used the EIT ITS p2+ system for measuring two different mixtures. In both cases the mixtures consisted of a conductive phase (water) and a non-conductive phase (transformer oil, silica sand). Because of the need for a sufficient difference between conductive and non-conductive phases the conductivity of water was increased by adding a small amount of sodium chloride $(\mathrm{NaCl})$. The conductivity of a strongly diluted solution of $\mathrm{NaCl}$ depends linearly [5] on the concentration of salt in water. This way its conductivity was raised to $0.8 \mathrm{mS} / \mathrm{cm}$ in the first experiment and to $1.5 \mathrm{mS} / \mathrm{cm}$ in the second one.

The container used for the experiment was a cylindrical vessel with four sets of sixteen electrodes each (thus giving us four independent EIT measuring planes), though for both following experiments we used only three. The mixing process also took place in this vessel using a Rushton turbine [6] made of plastic on a long plastic-composite shaft (see Figure 1). The vessel is equipped with a lid, which prevents aeration of the mixture during the mixing process. This is very important, because the media measured are truly only the two phases we introduced and the experiment is not invalidated by the generation of air vortex or air bubbles. With the lid closed the total volume of the measuring vessel is 6.361 .

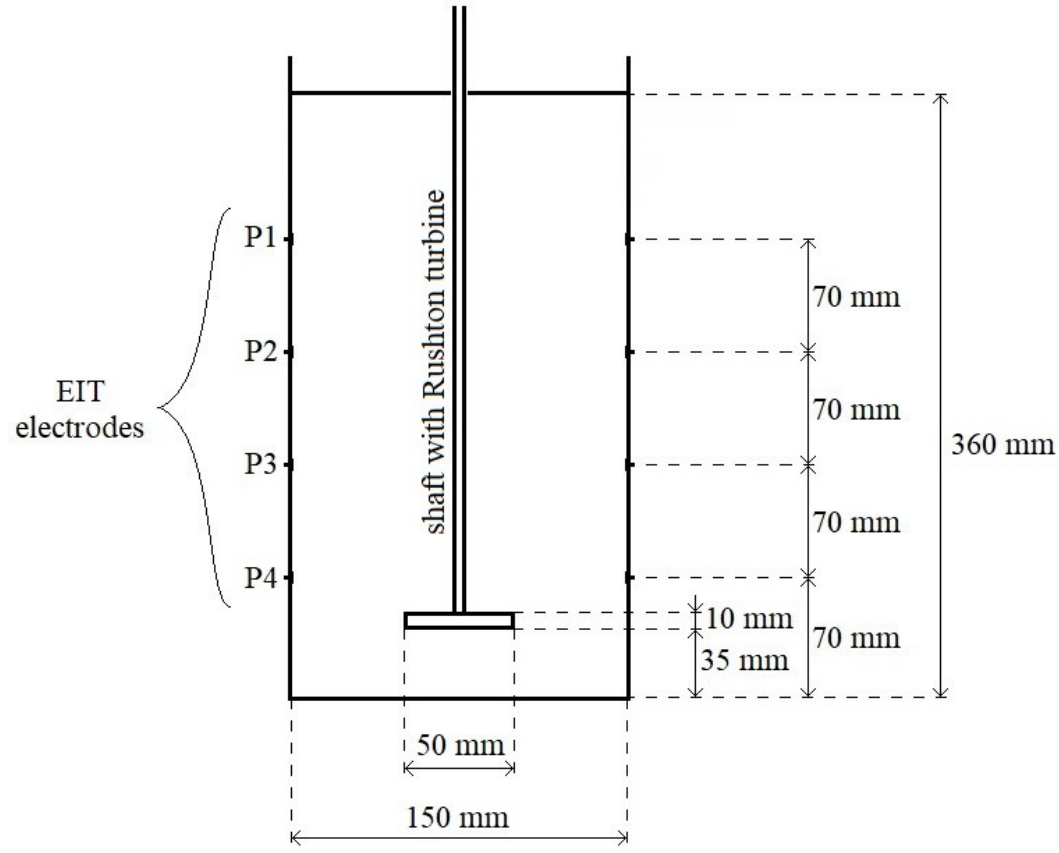

Fig. 1. Diagram of the measuring vessel with the Rushton turbine and EIT electrodes.

The materials of the shaft and turbine were chosen intentionally non-conductive, so the EIT analysis software would be able to mask their presence, thus ensuring that the measurement results would show only the two mixing phases. 


\section{Experiments}

The following two experiments were chosen to verify the theoretical formula (1). They also serve to prove the viability of ITS p2+ tomography system for measuring and analysing of mixing processes.

\subsection{Mixing water and oil}

For the first experiment we poured 0.71 of transformer oil into the measuring vessel and the rest of the space was filled by water with its conductivity increased to $0.8 \mathrm{mS} / \mathrm{cm}$. This gave us $11.1 \%$ of transformer oil in the whole volume of the measuring vessel. The EIT measurement was then started synchronously with the turbine. This provided the data from the whole mixing process from the very beginning until the homogenous mixture was reached. According to [7] while using Rushton turbine at speeds of several hundred rotations per minute, the mixed liquid should reach homogenous state after less than a minute. In our case the speed of the motor connected to the turbine was set to 760 RPM. The measurement was carried out through the point when the average conductivity in each measured plane became constant and was continued for another two minutes to assure homogenous conductivity distribution.

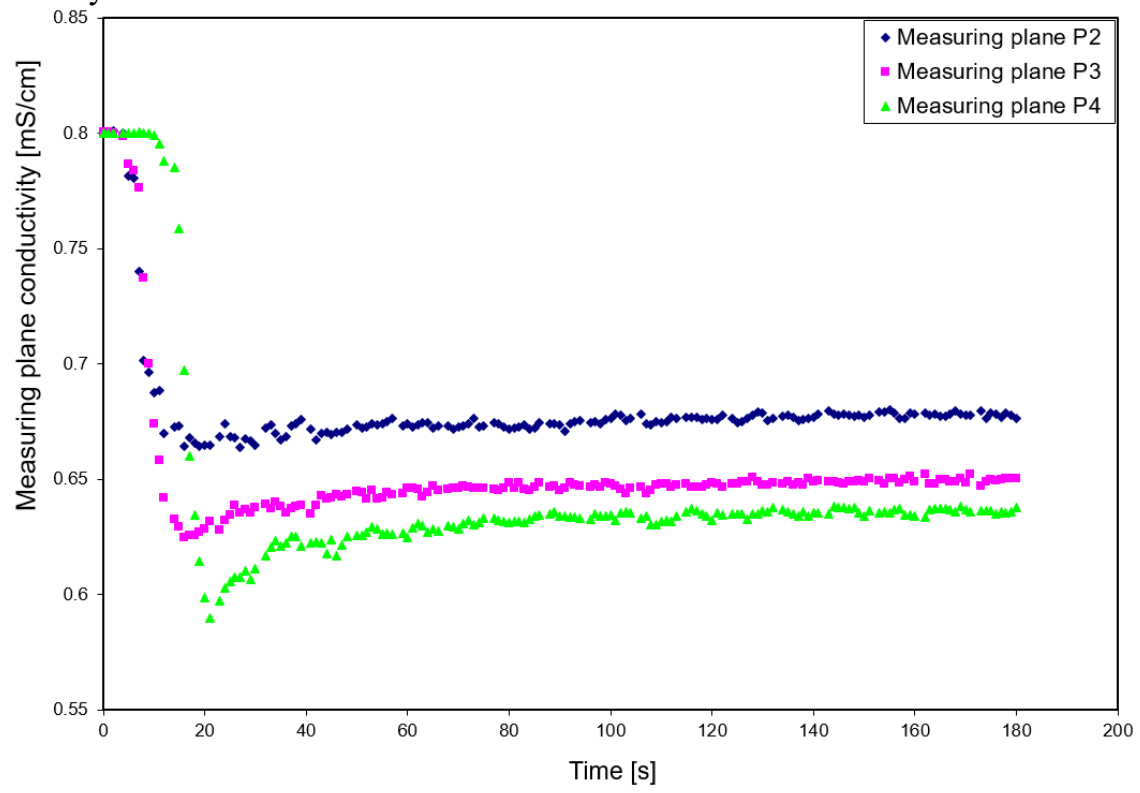

Fig. 2. Graph of time development of average conductivity in each measuring plane for the mixing process of water and transformer oil.

The mixing process was initiated by starting the turbine and simultaneously the measurement was started. This can be seen in Figure 2, which shows the time development of average conductivity in each measuring plane changing from the initial value of unmixed components to a final value representing a homogenous mixture. A series of photos showing the mixing process can be seen in Figure 3. 


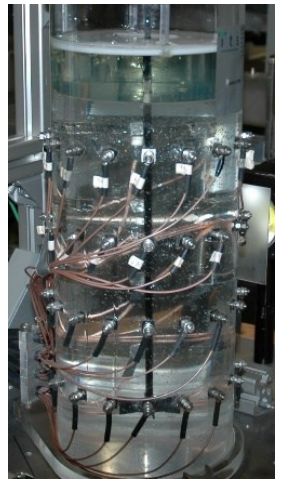

a)

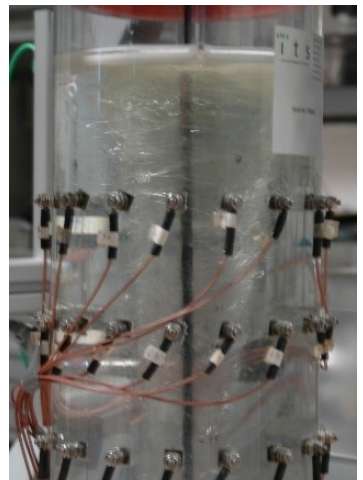

b)

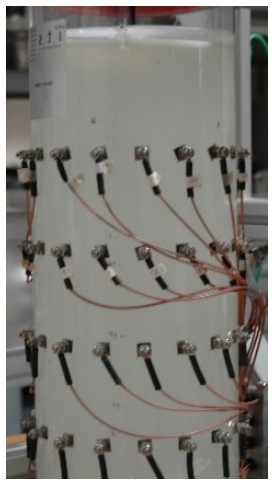

c)

Fig. 3. Photos of the measuring vessel a) with unmixed components (water - transformer oil), b) at the start of the mixing and c) at the point of homogenous mixture.

Since the transformer oil has lower density than water and at the beginning of the experiment it was all on top (see Figure 3a). This also affected the time of homogenisation as can be clearly seen from Figure 2, since the conductivity values in plane 2 are the first to become constant and the values in plane 4 are the last.

Second fact seen in Figure 2 is that after 35 seconds conductivity values in all three measuring planes become constant. The difference between them, though slight, is noticeable and it is caused by the dynamic equilibrium of forces affecting the mixed liquids (buoyancy, gravity and forces originating from the turbine caused vortex).

By using the average value of the three conductivities to represent the whole volume and putting it into the formula (1) we get the volume ratio $\varepsilon=0.12$ (nonconductive phase / conductive phase). From the initial conditions of the experiment we know the real volume ratio of the phases was $0.71: 5.661$, which equates to $\varepsilon^{\prime}=0.124$. This shows a perfect agreement.

\subsection{Mixing water and sand}

In the next experiment we wanted to test the methodology in the case of mixing the conductive liquid phase with a non-conductive solid phase. This time the measuring vessel was first filled with water to a height of $340 \mathrm{~mm}$ (volume $6 \mathrm{l}$ ). This gave us a space of $20 \mathrm{~mm}$ from the water surface to the lid (volume 0.361 ). Next we poured in silica sand (with particle size of $0.6-1.2 \mathrm{~mm}$ ) so that the water level rose to the surface of the lid, thus filling the vessel completely. This way the sand took up $5.5 \%$ of the total volume of the vessel. The reason for using only half the volume for the second phase was the possible strain on the plastic turbine, if we were to use more silica sand. This could lead to either heavy abrasion or even breaking of the turbine. Through the addition of $\mathrm{NaCl}$ the conductivity of water was again adjusted to a higher value, this time to $1.5 \mathrm{mS} / \mathrm{cm}$. Because the difference in densities of the first and second phase is in this case much greater than in the first experiment we had to use higher turbine speed. For this purpose the speed of the turbine was set to 1530 RPM. The measuring method used was also slightly modified. This time the EIT was used to capture not only the process of mixing from start until relative homogeneity, but also the process of relaxation after the motor has been turned off (see Figure 4). This way we can watch the conductivity of the mixed medium affected by sand rising from the bottom of the vessel because of the vortex and also by the opposite process when the sand is settling down during the relaxation. 


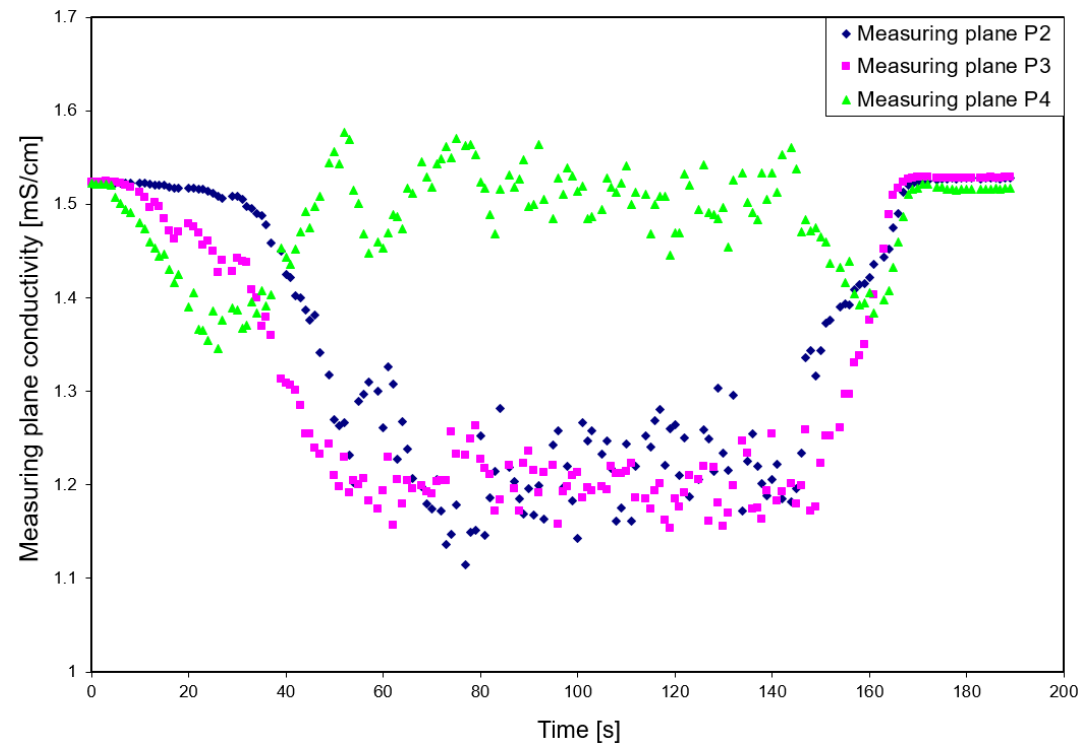

Fig. 4. Graph of time development of average conductivity in each measuring plane for the mixing process of water and silica sand.

A series of photos again shows the mixing of silica sand with water in the measuring vessel - see Figure 5.

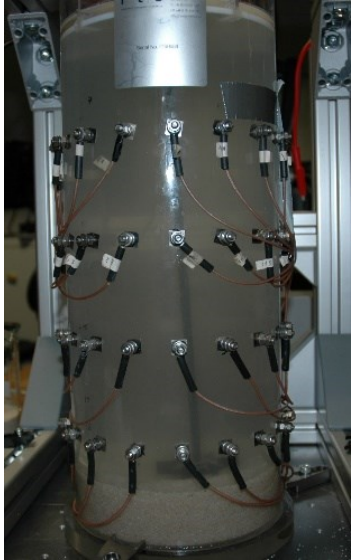

a)

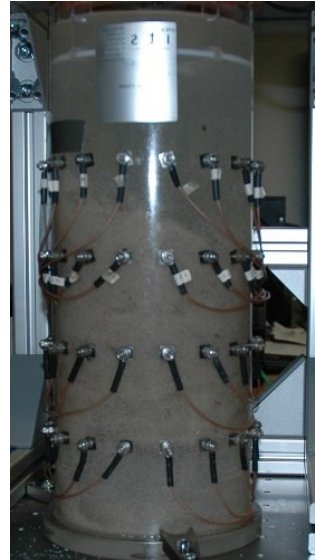

b)

Fig. 5. Photos of the measuring vessel a) with unmixed components (water - silica sand) and b) at the point of relatively homogenous mixture.

This time the second phase (silica sand) is much denser than water and at the beginning of the experiment it was all at the bottom of the vessel (see Figure 5a). This again affected the homogenisation times (see Figure 4), since the conductivity values in plane 4 are changing sooner and faster than the others. Unlike in the first experiment the conductivity in plane 4 returned very soon after the start of mixing to its initial value (on average). This was caused by the fact the silica sand rose all the way from the bottom of the vessel because of the vortex (in this experiment much stronger due to the higher RPM) affecting it. After the first 25 seconds almost all the sand left the measuring plane 4 and concentrated in between 
planes 2 and 3 as can be seen in Figure $5 \mathrm{~b}$. The overall fluctuations of the conductivity values were caused by the imperfect homogeneity and very strong turbulent flow.

By using the average value of conductivities in plane 2 and 3 to represent the mixture and putting it into the formula (1) we get the volume ratio $\varepsilon=0.14$. From the initial conditions of the experiment we know the real volume ratio of the phases was $0.361: 61$, which equates to $\varepsilon^{\prime}=0.06$. These values appear not to agree at all, but if we consider that the vast majority of the silica sand was concentrated in the area covered by measuring planes 2 and 3 , thus only it was present only in half of the total volume of the vessel. This corresponds with the fact the theoretically obtained value is approximately double of the real one. In which case we can consider the formula (1) to be still valid.

\section{Conclusion}

In this paper it has been shown that EIT system ITS p2+ is a viable tool for measuring mixing processes, where one of the mixed phases is considered non-conductive. It has also been shown, it works for both solids and liquids. This paper also brought experimental proof of an existing theoretical formula (1). The agreement of experimental results with calculated volume ratios is in the first experiment better than $96 \%$ and in the second experiment better than $85 \%$, which can be considered to be very good.

Tomography measuring methods are commonly used to view or analyse processes taking place inside objects. From the results of both described experiments it can be seen, the EIT provides more information and in greater detail about the examined processes than many other methods. Optical methods are usually used with great success for extremely precise measurements and observation of flows and possibly mixing of phases. However in our case they would fail, because the mixed materials used in our experiments are optically nontransparent. At much smaller volume ratios of mixed phases optical measuring methods (like shadowgraph method or particle/speckle image velocimetry [8]) may suddenly become viable, but this paper proves, the tomography measuring methods and namely the used EIT excel in these difficult conditions at great volume ratios of mixed phases.

Research of observing the mixing processes using EIT continues on the Technical university of Liberec in Czech Republic.

The Authors gratefully thank to the support of Grant Agency of the Czech Republic (GA ČR) 1620175S Local turbulent energy dissipation rate in dispersion systems, and LO1201 co-funding from the Ministry of Education, Youth and Sports as part of targeted support from the „National Programme for Sustainability I".

\section{References}

1. F. Dickin, M. Wang, "Electrical resistance tomography for process applications" in Meas. Sc. and Tech., 7, IOP Publishing, (1996)

2. A. P. Calderón, "On an inverse boundary value problem" in Comp. and App. Math., 25, Petropolis, (2006)

3. D. S. Holder, Electrical Impedance Tomography: Methods, History and Applications. London: Institute of Physics, (2004)

4. M. Liu, T. Wang, W. Yu and J. Wang, "An electrical conductivity probe method for measuring the local solid holdup in a slurry system" in Chem. Eng. J., 132, Elsevier, (2007)

5. A. Kay Smith, R. A. Gortner, "The Electrical conductivity of mixed salt solutions" in $J$. of Phys. Chem., 37 (1932) 
6. D. Jašíková, M. Kotek, V. Kopecký, “Time Resolved PIV Measurement of Fluid Dynamics in Agitated Vessels" in Proceedings of SPIE. USA : SPIE (2015)

7. D. Jašíková, M. Kotek, B. Kysela, V. Kopecký, "Experimental Identification of the Flow Vortex Structures Generated in the Agitated Vessels“ in Rec. Adv. in Mech. Eng., 11, Italy: World Academy of Science, (2014)

8. D. Jašíková, B. Kysela, J Konfršt, R. Šulc, "Estimation of turbulence dissipation rate by Large eddy PIV method in an agitated vessel" in EPJ Web of Conferences. France: EDP Sciences, (2015) 